Please note that this is not the final peer-reviewed version of this manuscript. Please do not cite this version. The final peer-reviewed version is in press at Psychological Science. This preprint will be updated with the doi for the peer-reviewed, published article when available.

See: Smith, A. M., Willroth, E. C., Gatchpazian, A., Shallcross, A.J., Feinberg, M., \& Ford, B. Q. (in press). Coping with health threats: The costs and benefits of managing emotions.

Psychological Science.

\title{
Coping with health threats: The costs and benefits of managing emotions
}

\author{
Angela M. Smith ${ }^{1}$, Emily C. Willroth ${ }^{2}$, \\ Arasteh Gatchpazian ${ }^{1}$, Amanda J. Shallcross ${ }^{3}$, Matthew Feinberg ${ }^{4}$, \\ \& Brett Q. Ford ${ }^{1}$
}

${ }^{1}$ Department of Psychology, University of Toronto

${ }^{2}$ Department of Medical Social Sciences, Northwestern University Feinberg School of Medicine

${ }^{3}$ Department of Population Health, New York University School of Medicine

${ }^{4}$ Rotman School of Management, University of Toronto

Correspondence regarding this article should be addressed to: Brett Ford, University of Toronto, Department of Psychology, 1265 Military Trail, Toronto, ON, M1C1A4. Email:

\section{Brett.Ford@utoronto.ca.}

Research was funded by an Insight Grant awarded to B.Q.F. from the Social Science and Humanities Research Council, a PEACE Grant awarded to B.Q.F. and A.J.S. from the Mind \& Life Institute, and a University of Toronto COVID-19 Student Engagement Award awarded to A.S. and A.G.

Author's Contribution: BQF, AMS, AG, AJS, and MF designed the study; ECW analyzed data; AMS, ECW and BQF wrote the first draft. All authors contributed to the analysis plan and provided substantive revisions. 


\begin{abstract}
How people respond to health threats can influence their own health and, when facing communal risks, even their community's health. We propose that people commonly respond to health threats by managing their emotions with cognitive strategies like reappraisal, which can reduce fear and protect mental health. However, because fear can also motivate health behaviors, reducing fear may also jeopardize health behaviors. In two diverse U.S. samples $(\mathrm{N}=1,241)$ tracked across three months, sequential and cross-lag panel mediation models indicated that reappraisal predicted lower fear about an ongoing health threat (COVID-19), and in turn, better mental health, but fewer recommended physical health behaviors. This trade-off was not inevitable, however: using reappraisal to increase socially-oriented positive emotions predicted better mental health without jeopardizing physical health behaviors. Examining the costs and benefits of how people cope with health threats is essential for promoting better health outcomes for individuals and communities.
\end{abstract}

Keywords: emotion regulation, reappraisal, fear, positive emotions, health behaviors, mental health, COVID-19 


\section{Statement of Relevance}

Health outcomes--both individual and communal--are influenced by how people respond to health threats. Commonly, people respond to these threats by regulating their emotions, which can reduce fear and thereby protect mental health. However, because fear can motivate effective health behaviors, reducing fear may also jeopardize the physical health of individuals and their communities. Considering the COVID-19 pandemic as a salient health threat, the present research indicates that although successfully using the emotion regulation strategy of reappraisal predicted lower fear and in turn, better mental health, it also predicted lower physical healthprotective behaviors, putting individuals and their communities at risk. However, reappraisal was also used to cultivate positive emotions (e.g., gratitude, inspiration), thereby predicting better mental health without jeopardizing physical health behaviors. These findings are among the first to point to the perils (in addition to the promise) of using emotion regulation in the face of health threats. 


\section{Coping with health threats: The costs and benefits of managing emotions}

The way people respond to health threats not only affects their own health but the health of others. This is particularly true for infectious diseases, where one person's actions can have downstream impacts for the broader community. Thus, understanding what underlies people's reactions to health threats and harnessing this knowledge to promote adaptive responses to threat can be fundamental to improving individual and community well-being.

At the heart of how people respond to health threats is the emotion of fear (Folkman \& Greer, 2000). Although fear feels unpleasant, it motivates people to protect their physical health by engaging in recommended health behaviors (Harper, Satchell, Fido, \& Latzman, 2020). However, precisely because fear is unpleasant, people are often -- understandably -- motivated to reduce fear (Gross, 2015). Reducing one's day-to-day experience of fear should come with important benefits for longer-term mental health, especially in the face of ongoing health threats (Dieng et al., 2016). But because reducing fear also reduces the motivation it provides, people who successfully reduce fear about a health threat may also be less likely to engage in physical health behaviors that protect not only themselves but also their communities. We examine this dilemma within the present research, focusing on a commonly used form of emotion regulation that can effectively reduce fear: cognitive reappraisal, or reframing situations to change their emotional impact (Gross, 2015).

The present research bridges affective science with health psychology to generate novel predictions about how emotion regulation can influence health and illness. In doing so, this research makes three theoretical contributions. (1) The scientific community does not know whether the very forms of emotion regulation designed to help people cope with the stress of health threats can further jeopardize people's physical health outcomes. We address this core gap 
by examining the effects of reappraisal on people's real-world health behaviors in the context of a health threat, providing evidence for the underlying mechanisms of this effect. (2) The present research examines a crucial trade-off of emotion regulation: Although using reappraisal to reduce fear may predict fewer health behaviors, it should also predict better mental health -- an unfortunate dilemma that puts the people in a difficult situation as they attempt to cope with the stress of a health crisis. By bringing this trade-off to light, this work can help researchers in both the affective sciences and health fields update how they conceptualize the benefits and costs of reappraisal. (3) We investigate an alternative pathway through which the trade-offs of reappraisal could be avoided. Namely, although much research examining emotion regulation in the context of stress focuses on the role of negative emotion, we highlight the crucial role of positive emotion: Theorizing that positive emotion's unique features may help avoid the trade-offs of using reappraisal to decrease fear, we predict that certain positive emotions should provide emotional relief that can protect mental health without carrying the same costs to health behaviors that can jeopardize physical health. By considering this under-studied target of reappraisal, we are able to suggest a potential solution for the drawbacks of reappraisal when managing threats to one's physical health. To test these predictions, we examined two large, diverse U.S. samples, across ten waves of longitudinal data during the COVID-19 pandemic -- a powerful threat to both physical and mental health.

\section{The Role of Fear in Responding to Health Threats}

When people face significant threats to their health, they often experience fear (Folkman $\&$ Greer, 2000). Consistent with functional accounts of emotions (Frijda, 1986), this fear serves an important purpose by motivating people to engage in behaviors that can protect health (Mayne, 1999). For example, people who felt afraid of breast cancer were more likely to 
consider procedures to reduce their risk of developing breast cancer (Tesson et al., 2017); those who felt afraid of sexually transmitted infections were more willing to take a screening test (Shepherd \& Smith, 2017); and parents who felt afraid of their child contracting measles were more likely to have their child vaccinated (Feigelman, 1993). This evidence suggests that although fear is unpleasant, it can effectively motivate people to engage in behaviors that protect their physical health -- and even their community's health, in the case of communal health risks.

As useful as fear can be for motivating physical health behaviors, fear is highly unpleasant (Russell \& Barrett, 1999) and can, over time, negatively impact people's mental health (Kotov et al., 2010). To protect themselves from undesirable or painful emotions, people often use emotion regulation strategies to reduce these emotions. One particularly common regulation strategy -- even when facing health threats (Moskowitz et al., 1996) -- is cognitive reappraisal (Ford et al., 2017). Because reappraisal involves changing one's perspective on the nature of the threat one is facing (Uusberg et al., 2019), it can powerfully shape people's emotional experiences. For example, reappraisal can be used to minimize or downplay the severity of a threatening situation. Such potent shifts to one's perspective can in turn effectively reduce fear.

\section{The Benefits and Costs of Reappraisal}

Reappraisal is typically considered so helpful for managing emotions that it is viewed as a gold standard regulation strategy and has even become popular in various fields outside of psychology (see Ford \& Troy, 2019). In many ways, this popularity is warranted: Reappraisal has been consistently linked to better mental health outcomes when used in general (Aldao et al., 2010) and when used in the context of health threats. For example, using reappraisal to cope with health-related fear predicted lower depression and anxiety in HIV-positive men (Kraaij et al., 
2008), in people undergoing infertility treatment (Kraaij et al., 2010), and in newly-diagnosed cancer patients (Peh et al., 2016; Wang et al., 2013). Based on these findings, one might conclude that reappraisal should be widely used by individuals facing health threats.

In addition to its benefits, however, reappraisal also comes with drawbacks (Ford \& Troy, 2019). By reducing emotions that drive functional behavior, reappraisal can impair motivation to take appropriate action when action is needed most (Feinberg et al., 2020; Troy et al., 2013; van't Wout, et al., 2010), even when that action can protect one's community (Ford et al., 2019). In the context of health threats, using reappraisal to reduce fear (e.g., by minimizing the severity of the threat) should reduce the motivation that fear provides to avoid these health threats. Although these drawbacks have not yet been examined in the high-impact domain of health threats, one recent study provides suggestive evidence: in line with our reasoning, people who used reappraisal to reduce negative emotions in the face of threat-inducing health messages were less convinced by those messages (i.e., anti-binge drinking messages; Doré et al., 2019) -- a result that strongly suggests such people would be less likely to follow the health behaviors recommended by the message.

Given the influence that health behaviors can have on social networks (Christakis et al., 2008), using reappraisal to reduce fear of a health threat may affect not only individuals' physical health outcomes, but also community outcomes. For example, when facing an infectious disease, less engagement in health behaviors (e.g., hand-washing) can exacerbate the spread of disease. Taken together, prior theory and indirect evidence suggests that using reappraisal to reduce fear in the face of physical health threats may come with a crucial trade-off: greater mental health but lesser behaviors that protect physical health of individuals and communities. 


\section{An Alternative to Reducing Fear in the Face of Health Threats}

Using reappraisal can help reduce fear in the face of health threats and, in turn, protect mental health while also jeopardizing physical health behaviors. But is this trade-off inevitable? Reducing fear is not the only way to feel better and striving to avoid fear can be problematic in itself (Mennin, 2005). Fortunately, reappraisal can also be used to increase the experience of positive emotions (Shiota \& Levenson, 2012), which can promote mental health (Jans-Beken et al., 2019) and help avoid the trade-offs associated with reducing fear (Cook \& Chater, 2010). Even in the face of health threats, reappraisal can be used to reconsider such situations as opportunities for growth, meaning, and connection (Folkman, 1997), and can thereby enhance positive emotional experiences without necessarily minimizing the threat as less severe or consequential. Given this, using reappraisal to cultivate positive emotions is unlikely to interfere with health behaviors. In the high impact context of communal health threats -- when health behaviors are not only self-protective but also community-protective -- positive emotions that are oriented towards other people (e.g., gratitude, love, and admiration) may even promote preventative physical health behaviors that can keep the self and others safe.

\section{Current Investigation}

The current investigation bridges affective science with health psychology to test whether using reappraisal to reduce fear evokes a trade-off between mental health and physical healthprotective behaviors. The COVID-19 outbreak provided a unique opportunity to examine this trade-off in the context of a salient and fear-provoking communal health threat. We expected that many people would feel fearful in response to the outbreak, and many would use reappraisal to manage their emotions about the outbreak. We expected that successful reappraisal would predict lower fear and in turn better mental health (i.e., reduced depression and anxiety 
symptoms) but would also predict less engagement in preventative health behaviors recommended by the U.S. Centers for Disease Control (CDC; e.g., mask-wearing, socialdistancing). We also tested an alternative pathway that may avoid these trade-offs: whether successful reappraisal can also help people cultivate positive emotions towards other people (e.g., gratitude, inspiration) in the context of the outbreak, and in turn predict greater mental health without jeopardizing engagement in recommended health behaviors.

To test these questions, we leveraged two multi-wave longitudinal samples across a real unfolding health crisis. We recruited two large U.S. samples (total $N=1,241$ ) that were diverse in racial and ethnic identity, political orientation, and socioeconomic status and analyzed ten waves of data collection across three months, beginning in late February 2020, before COVID-19 was declared a pandemic. This design enabled us to capture the time period when the pandemic first emerged and when it became a global crisis, covering this event more comprehensively than research that typically begins after a crisis has occurred. Given the structure of these data, the present research is uniquely able to address how reappraisal predicts change in negative emotion (and positive emotion), and in turn, change in health behaviors and mental health over time. Specifically, we compared results across two types of mediation models: first capitalizing on our longitudinal design with sequential mediation models (Sample A and B) and then providing a stronger test of causality with cross-lag panel mediation models that control for previous levels of each variable (Sample A). Although true causality cannot be tested using observational data, cross-lag panel models test Granger causality, a statistical concept that in this case means reappraisal (the hypothesized cause) precedes and predicts emotions (the hypothesized mediator), which in turn precede and predict health behaviors and mental health (the hypothesized outcomes) (Cain et al., 2017). This is a key strength of the present longitudinal 
design, given that we could not ethically manipulate factors expected to reduce health-protective behaviors that are vital for slowing the spread of COVID-19.

\section{Method}

\section{Participants}

Participants were recruited from Amazon's Mechanical Turk -- an online platform that allowed us to rapidly collect time-sensitive data from diverse participants. Participants were required to be residents of the United States, have a 95\% approval rating, and have completed at least 100 HITs on the Mechanical Turk platform. On an a priori basis, data were excluded from measurement occasions where a participant did not pass all of the provided attention checks, provided incomplete data, or took the survey more than once (see Supplemental Online Materials for more detail about data quality checks and exclusions). In all models, the sample size for both samples exceeded best-practice recommendations of 20 participants per parameter estimate, and a minimum total sample size of 200 (Kline, 2015).

Sample A. Sample A included 742 participants originally recruited in February 2020, and the number of participants included in each analysis ranged from 518 to 696 based on different missing data patterns for the sequential mediation and cross-lag panel models. Participants who were included in at least one analysis ranged from 18-75 years old $(\mathrm{M}=36.96, S D=11.18)$ and were $53 \%$ women, $44 \%$ men, $3 \%$ other gender or did not report gender; $30 \%$ African or African American, 22\% East Asian or East Asian American, 34\% European American/White/Caucasian, 9\% South Asian or South Asian American, 4\% other racial or ethnic identities; and 50\%

Democrats, 16\% Republicans, and 30\% Independents. Participants in Sample A were recruited to be relatively diverse with respect to ethnic and racial identity. 
Sample B. Sample B is completely separate from Sample A and included 842 participants originally recruited in February 2020, and 545 participants provided data for all of the timepoints and measures used in the sequential mediation models (cross-lag panel models comparable to Sample A were not possible in Sample B). The participants included in the present analyses ranged from 20 to 81 years old $(\mathrm{M}=43.22, S D=13.16)$ and were $50 \%$ women, $49 \%$ men, $1 \%$ other gender or did not report gender; $6 \%$ African or African American, 5\% East Asian or East Asian American, 83\% European American/White/Caucasian, 3\% South Asian or South Asian American, 3\% other race or ethnic identities; and 51\% Democrats, and 49\% Republicans. Participants in Sample B were recruited to be relatively diverse with respect to political ideology.

\section{Procedure}

The present research is part of a large longitudinal study aimed at understanding how psychosocial factors influence individuals' responses to the COVID-19 pandemic. Data from the first ten waves of data collection are included in the present study. Figure 1 displays the temporal sequencing of each wave of data collection, including when constructs were assessed for Sample A and B. Sample A participants were originally recruited for a longitudinal study centered on coping with the stress of the COVID outbreak; these participants were invited to complete a baseline survey in late February (Time 1; T1). Participants who passed attention checks at T1 were invited to participate in future waves of the study. Sample B participants were originally recruited for a different longitudinal study centered on coping with the stress of politics; these participants were invited to complete a different baseline survey in mid-February that did not include any COVID-related questions. These participants were invited into the COVID-focused study at Time 2 (T2) and participants who passed attention checks at T2 were invited to all future waves of the COVID-focused study. 
After the baseline survey (T1), all participants were invited to complete longer monthly surveys in late March, late April, and late May (T2, T3, T4) and shorter weekly surveys in March (T1a, T1b, and T1c; Sample A only) and April (T2a, T2b, and T2c). The longer monthly surveys ranged from $\sim 30$ to 35 minutes and participants were compensated between $\$ 4.50$ and $\$ 5.00$ for their time depending on the length of the survey. The shorter weekly surveys ranged from 1 minute to 20 minutes and participants were compensated between $\$ 0.25$ and $\$ 3.50$ for their time depending on the length of the survey (effective median hourly rate $\sim \$ 9$ USD). All procedures were approved by the ethics board at [Masked for review] (protocol \#33962).

\section{Figure 1.}

Summary of measurements included in the present analyses, the timeline along which these measures were collected for Sample A and B, and the broader COVID-19 context, as represented by concurrent cumulative case numbers.

Study Measurement Summary, Timeline, and Broader COVID-19 Context:

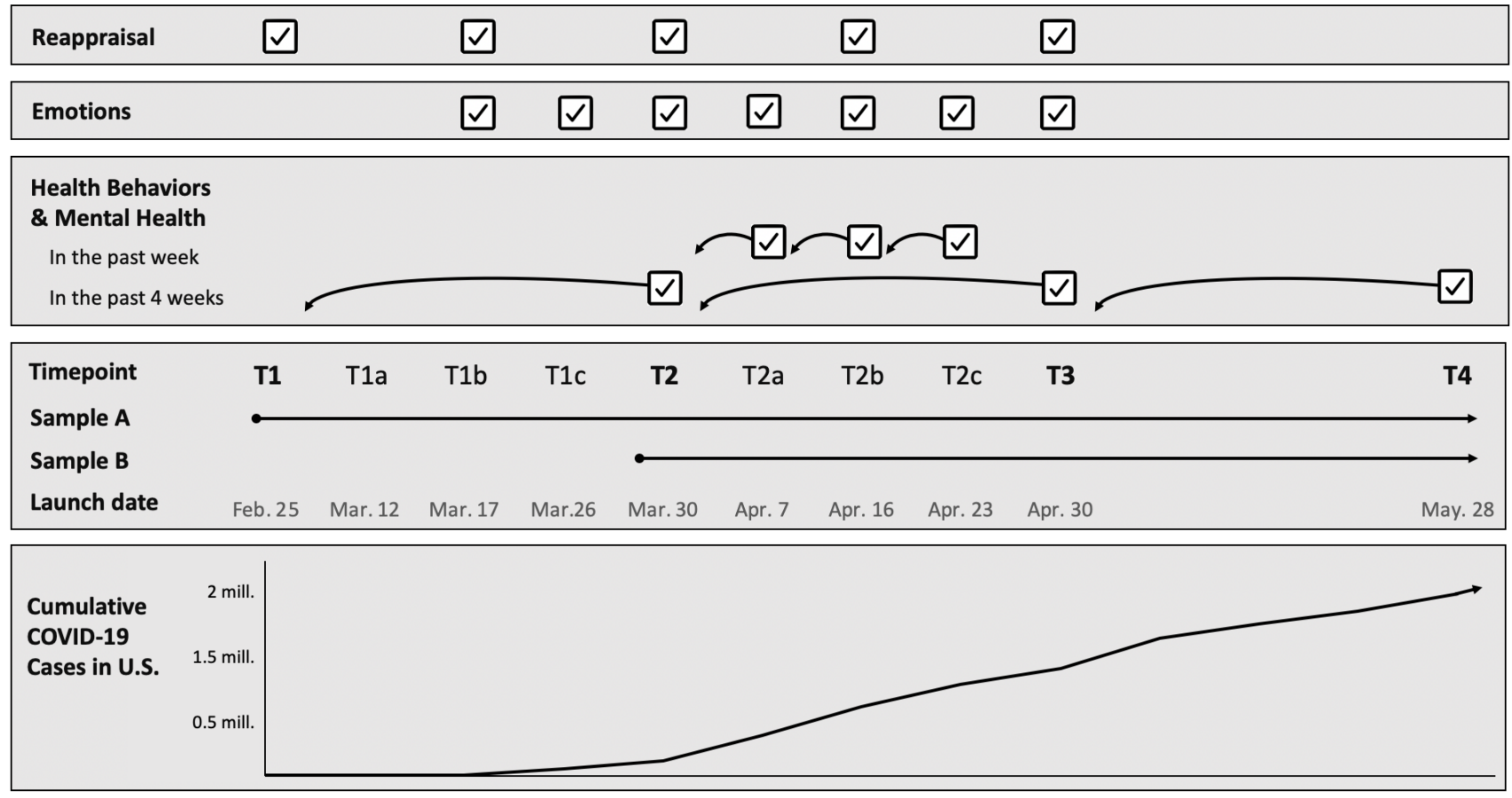

Note. Sample B completed a T1 survey in mid-February, but it did not include any measures related to COVID (and as such, is not included in any analyses reported in the present paper). 


\section{Measures}

Reappraisal. Participants completed separate ratings for how frequently they used reappraisal and how successfully they used reappraisal when feeling stressed about the COVID19 outbreak at T1, T1b, T2, T2b, and T3 in Sample A and at T2, T2b, and T3 in Sample B. First, participants rated four reappraisal frequency items on a scale from 0 (I never try this) to 6 (I always try this), with items like "When you want to feel less negative emotion about the recent coronavirus outbreak (such as anxiety, disgust, or frustration), do you try to change the way you're thinking about the outbreak?" and "When you want to feel more positive emotion about the outbreak (such as hope or relief), do you try to change the way you're thinking about the outbreak?" Then, participants rated their reappraisal success on a scale from 0 (I am definitely unable to do this) to 6 (I am definitely able to do this), with items like "When you want to feel less negative emotion about the recent outbreak (such as anxiety, disgust, or frustration), are you able to change the way you're thinking about the outbreak, if you try?" and "When you want to feel more positive emotion about the outbreak (such as hope or relief), are you successful at changing the way you're thinking about the outbreak, if you try?" (all items for both reappraisal frequency and success are reported in the Supplementary Online Materials). We computed a mean reappraisal frequency composite and a mean reappraisal success composite at each timepoint $($ alpha $=.88-.93)$. For illustrative purposes, we report descriptive statistics for reappraisal frequency below. However, for our inferential analyses, we focus on reappraisal success, given that emotion regulation successes and attempts are conceptually and empirically distinct (Ford et al., 2017), and it is the successful use of a given strategy that should impact emotional outcomes, not merely the number of times they attempt the strategy. 
Emotions. We assessed emotions about the COVID-19 pandemic at each timepoint. Participants responded to the prompt "In the past day or two, to what extent have you felt these emotions when thinking about the outbreak?" Response options ranged from 0 (Not at all) to 6 (Extremely). Socially-oriented positive emotions were introduced beginning at T1b, and thus earlier measurements of emotions were not used in the present analyses. We assessed up to 24 emotion items at each timepoint, but on an a priori basis, focused on the two item triplets related to fear and three item triplets related to social positive emotions for the present study. To assess fear, we used the following item triplets: "worried, nervous, fearful," and "panicked, alarmed, freaked out" (alpha $=.81-.88)$. To assess socially-oriented positive emotions, we used the following item triplets: "love, closeness, trust," "inspired, uplifted, elevated," and "grateful, appreciative, thankful"' (alpha $=.80-.86)$.

Preliminary analyses revealed that although fear and socially-oriented positive emotions are related, they are also empirically distinct: In a multilevel model that predicted sociallyoriented positive emotions from fear, there was a modest negative association between fear and social positive emotions at the between-person level: Sample A: $\mathrm{b}=-.23, p<.001$; Sample B: $\mathrm{b}$ $=-.23, p<.001$ (these variables were $\mathrm{z}$-standardized here so that these coefficients can be roughly interpreted as betas). To examine the specificity of the link between fear and health behaviors, we also considered other negative emotions (i.e., the relatively social negative emotions of guilt and shame); and to examine the specificity of the link between sociallyoriented positive emotions and health behaviors, we also considered other positive emotions (i.e., the relatively less social emotions of happiness and amusement). These results (reported in the Supplementary Online Materials) indicate that the pattern of results reported in the primary text are specific to fear and socially-oriented positive emotions: although reappraisal predicted lower 
negative and greater positive emotions broadly construed (consistent with the broad hedonic benefits of reappraisal), only fear and socially-oriented positive emotions were consistent predictors of health behaviors (consistent with a functional account of particular emotions).

Health Behaviors. We assessed the frequency with which participants engaged in COVID-19 preventative health behaviors recommended by the CDC. In the weekly assessments, beginning with T2a, participants rated how often they engaged in each of four health behaviors over the past week (i.e., practicing hygiene, wearing a face mask, social distancing, and social isolation; see Supplement for exact item wording). In the monthly assessments, beginning with T2, participants rated how often they engaged in each of five health behaviors over the past month (i.e., practicing hygiene, wearing a face mask, sanitizing surfaces, social distancing, and social isolation). Response options ranged from 0 (I did not do this) to 4 (I very often or always did this). We computed a mean composite across the four (or five) health behaviors at each timepoint (alpha $=.60-.80)$.

Mental Health Symptoms. We assessed two aspects of mental health: depressive symptoms and anxiety symptoms. In the weekly assessments, beginning at T2a, we used single item measures to assess depression and anxiety in the past week: "This week, I felt depressed" and "This week, I felt anxious." Response options ranged from 0 (I do not agree with this at all) to 6 (I completely agree with this). We computed the mean of the two items at each timepoint $($ alpha $=.79-.82)$. In the monthly assessments, we used the Center for Epidemiological Studies Depression Scale (Radloff, 1977) to assess depression symptoms over the past four weeks and the Hospital Anxiety and Depression Scale (Snaith, 2003) to assess anxiety symptoms over the past four weeks. To create an aggregate score, we first z-standardized each scale score at each 
timepoint and then computed a mean mental health composite of the two z-scored variables at each timepoint (alpha $=.91)$.

\section{Analytic Strategy}

To examine the effects of reappraisal on emotions, and in turn on health behaviors and mental health, we used two types of mediation models: '2-1-2' sequential mediation models and cross-lag panel mediation models. Note that all reported bs for these analyses are unstandardized. Sequential Mediation. In the first set of mediation models, we used sequential mediation, a form of longitudinal mediation (Cain et al., 2017). Longitudinal mediation is preferable to crosssectional mediation, because it allows time for the predictor to influence the mediator and for the mediator to influence the outcome (Maxwell \& Cole, 2007). On a priori basis, we chose T1b for our measure of reappraisal success in Sample A because this measurement occasion took place approximately one week after the World Health Organization declared COVID-19 a pandemic, allowing time for emotional responses to the pandemic and thus, emotion regulation to take place. For Sample B, we used the first measurement occasion at which reappraisal was assessed, T2. To provide parallel tests across similar time spans in the 2-1-2 sequential and cross-lag panel mediation models, we chose T4 for the outcome measures in both samples. We used all available measurement occasions of emotions between our reappraisal measure and our outcome measures. We used a '2-1-2' random effects sequential mediation model in which the predictor (reappraisal) and the outcome (health behaviors or mental health symptoms) were included at

level 2 (i.e., one observation per participant) and repeated measures of the mediator (emotions) were included at level 1 (i.e., multiple observations per participant consisting of one observation per measurement occasion), as described in Preacher et al., 2011 (see Figure 2). 


\section{Figure 2.}

Path diagram of 2-1-2 sequential mediation model, in which the predictor (reappraisal) and the outcome (health behaviors or mental health symptoms) were included at level 2 and repeated measures of the mediator (emotions) were included at level 1.

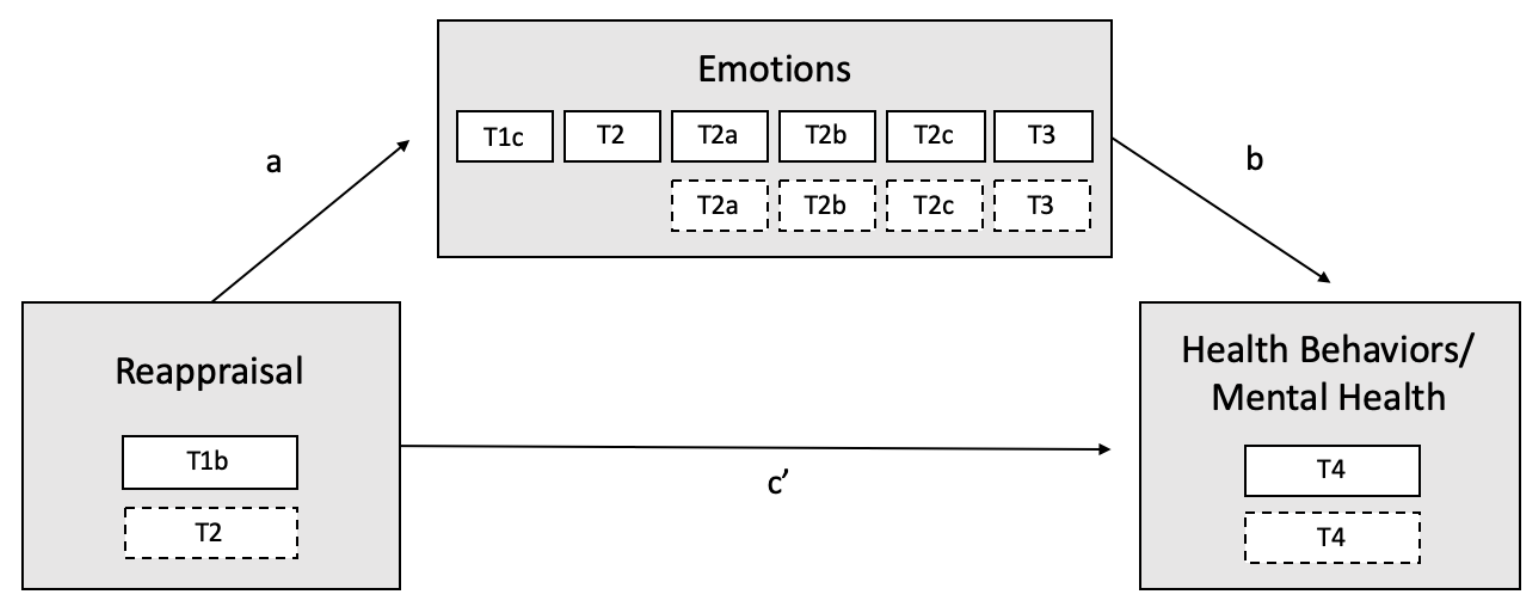

Note. Solid outlines represent the timepoints that were included in Sample A. Dashed outlines represent timepoints that were included in Sample B.

Cross-lag Panel Mediation. Although sequential mediation allows effects to take place over time, it does not account for previous values of the variables in the model. To address this limitation, we also tested cross-lag panel mediation models (Selig \& Preacher, 2009) in Sample A (requisite data was not available in Sample B because the use of reappraisal in response to COVID was not assessed until T2). Cross-lag panel models test the prospective effect of individual differences in a construct (in this case, reappraisal) on change in individual differences in another construct (in this case, emotions, health behaviors, and mental health). By accounting for previous values of the variables with autoregressive paths, cross-lag panel mediation provides a stronger test of causality relative to sequential mediation (Cain et al., 2017).

One challenge of lagged analyses is specifying a time lag that approximates the true causal lag (Selig \& Preacher, 2009). To address this, we tested two versions of a cross-lag panel mediation model which differed according to the specified time lag (see Figure 3). In the first model, we used a time-lag of approximately one-week in between measurement occasions. In the 
second model, we used a time-lag of approximately one-month in between measurement occasions. In the weekly model, the predictor (reappraisal) and the mediator (emotions) were always assessed at the same time as one another (at T1b, T2, and T2b) and the outcomes (health behaviors and mental health symptoms) were assessed approximately one week later (at T2, T2b, and T2c) because the measures used to assess the outcomes referred to health behaviors and mental health symptoms "in the past week". We used a similar approach for the monthly models: Reappraisal and emotions were assessed at the same time as one another (T1, T2, T3), and health behaviors and mental health symptoms were assessed one month later (T2, T3, T4) because the measures used to assess the outcomes referred to health behaviors and mental health symptoms “in the past month". This monthly model had one exception: Because socially-oriented positive emotions were not assessed at $\mathrm{T} 1$, the first wave of emotion measurements occurred at $\mathrm{T} 1 \mathrm{~b}$.

\section{Figure 3.}

Path diagram of cross-lag panel mediation model (in Sample A only).

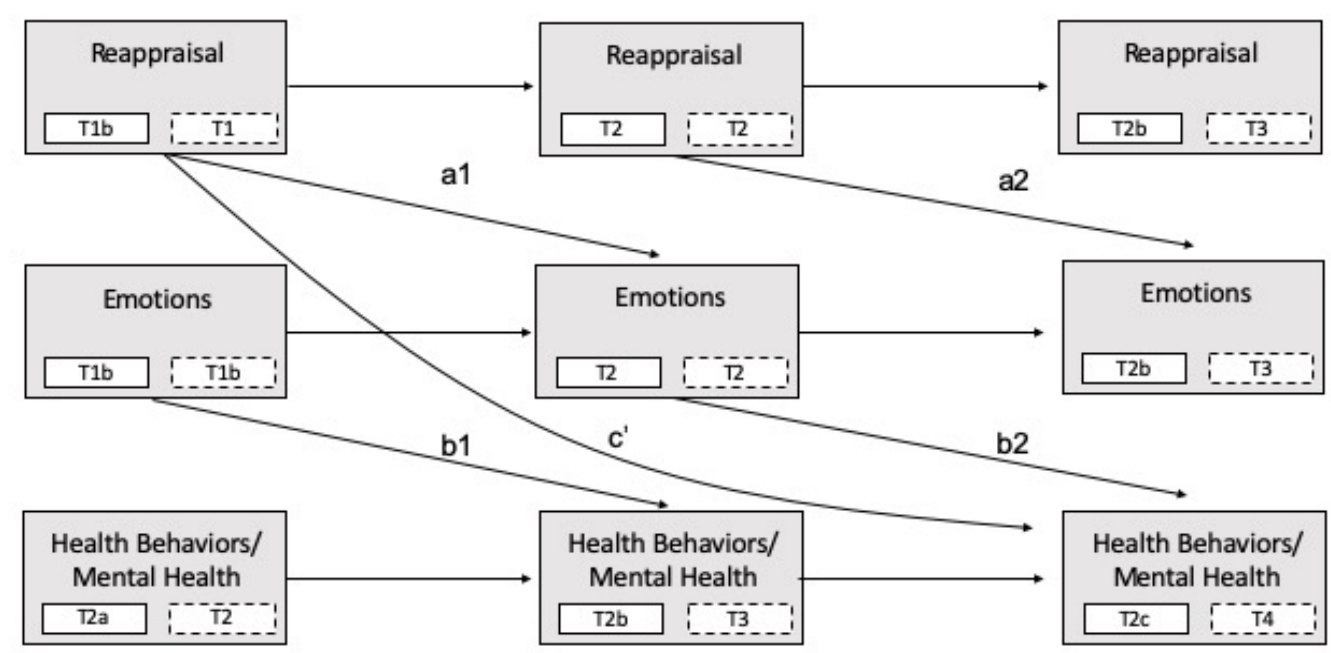

Note. Solid outlines represent the timepoints that were included in the 'weekly' models. Dashed outlines represent timepoints that were included in the 'monthly' models. Note that to estimate a single indirect effect, $\mathrm{a} 1$ and $\mathrm{a} 2$ were constrained to be equal and $\mathrm{b} 1$ and $\mathrm{b} 2$ were constrained to be equal. 


\section{Results}

Data and statistical code to reproduce all results is available at https://osf.io/y8kp3/?view_only=32bd0b2dc3a842a38e90218b031852a7. Descriptive statistics

for study variables are shown in Supplementary Table 1. Fear and socially-oriented positive emotions were common responses to the COVID-19 outbreak, with $80 \%$ to $94 \%$ of participants experiencing at least some fear and $89 \%$ to $97 \%$ of participants experiencing at least some socially-oriented positive emotions across measurement occasions and samples. Many people -$97 \%$ in both samples -- used reappraisal to at least some degree in response to emotions about the COVID-19 outbreak at the first assessment point. The average reappraisal frequency values (Sample A: $\mathrm{M}=3.13, \mathrm{SD}=1.33$; Sample $\mathrm{B}: \mathrm{M}=3.10, \mathrm{SD}=1.39$ ) corresponded to people using reappraisal 'sometimes' in daily life, on average, with wide individual variability. The average reappraisal success values (Sample A: $\mathrm{M}=3.47, \mathrm{SD}=1.39$; Sample B: $\mathrm{M}=3.37, \mathrm{SD}=1.48$ ) corresponded to people being "somewhat" to "moderately" successful, with substantial individual variation. These descriptive findings set the stage for examining how people's successful use of reappraisal predicts downstream emotional experiences, and in turn, health behaviors and mental health.

\section{Fear and Health Behaviors}

In the sequential mediation models (summarized in the top-half of Table 1), reappraisal predicted lower fear, which in turn predicted fewer health behaviors. The indirect effect of reappraisal on fewer health behaviors, mediated by fear, was statistically significant in both samples.

The cross-lag panel mediation models replicated the sequential mediation models: reappraisal predicted lower fear, which in turn predicted fewer health behaviors. The indirect 
effect of reappraisal on fewer health behaviors, mediated by fear, was marginally significant in the weekly model, and was statistically significant in the monthly model.

Table 1

Reappraisal Predicting Lower Fear and in Turn, Fewer Health Behaviors but Better Mental Health (i.e., Fewer Mental Health Symptoms)

\section{Predicting Health Behaviors}

\section{Sequential Mediation Model Paths}

\begin{tabular}{cccccccc}
\hline \multicolumn{3}{c}{ Sample A $(\mathrm{N}=696)$} & & \multicolumn{5}{c}{ Sample B (N=545) } \\
\hline $\mathrm{b}$ & $\mathrm{CI}$ & $\mathrm{SE}$ & $p$ & $\mathrm{~b}$ & $\mathrm{CI}$ & $\mathrm{SE}$ & $p$ \\
\hline-.309 & $-. .394,-.223$ & .044 & $<.001$ & -.479 & $-.554,-.404$ & .038 & $<.001$ \\
.129 & $.077, .182$ & .027 & $<.001$ & .165 & $.101, .229$ & .033 & $<.001$ \\
.022 & $-.033, .077$ & .028 & .430 & .064 & $.004, .124$ & .031 & .036 \\
-.040 & $-.060,-.020$ & .010 & $<.001$ & -.079 & $-.112,-.046$ & .017 & $<.001$ \\
\hline & Sample A Weekly (N=530) & & & Sample A Monthly (N=518) \\
\hline $\mathrm{b}$ & $95 \%$ CI & $\mathrm{SE}$ & $p$ & $\mathrm{~b}$ & $95 \%$ CI & $\mathrm{SE}$ & $p$ \\
\hline-.067 & $-.123,-.011$ & 0.029 & .019 & -.089 & $-.146,-.031$ & .029 & .002 \\
.026 & $.009, .042$ & 0.008 & .002 & .030 & $.008, .051$ & .011 & .008 \\
.009 & $-.019, .037$ & .014 & .517 & .020 & $-.016, .057$ & .019 & .275 \\
-.002 & $-.004, .000$ & 0.001 & .062 & -.003 & $-.005, .000$ & .001 & .045 \\
\hline
\end{tabular}

a path

Predicting Mental Health Symptoms

\section{Cross-lag Panel}

Mediation Model Paths:

a path

b path

c' path

Indirect effect $(a * b)$

\begin{tabular}{cccccccc}
\hline \multicolumn{3}{c}{ Sample A (N=696) } & \multicolumn{7}{c}{ Sample B (N=545) } \\
\hline $\mathrm{b}$ & $95 \%$ CI & SE & $p$ & $\mathrm{~b}$ & $95 \%$ CI & SE & $p$ \\
\hline-.311 & $-. .396,-.225$ & .044 & $<.001$ & -.479 & $-.554,-.403$ & .039 & $<.001$ \\
.441 & $.393, .489$ & .024 & $<.001$ & .513 & $.464, .563$ & .025 & $<.001$ \\
-.014 & $-.065, .037$ & .026 & .585 & -.019 & $-.065, .027$ & .024 & .415 \\
-.137 & $-.178,-.097$ & .021 & $<.001$ & -.246 & $-.291,-.200$ & .023 & $<.001$ \\
\hline & Sample A Weekly (N=530) & & & Sample A Monthly (N=518) & \\
\hline $\mathrm{b}$ & $95 \%$ CI & $\mathrm{SE}$ & $p$ & $\mathrm{~b}$ & $95 \%$ CI & $\mathrm{SE}$ & $p$ \\
\hline-.067 & $-.123,-.010$ & .029 & .020 & -.087 & $-.144,-.029$ & .029 & .003 \\
.091 & $.046, .135$ & .023 & $<.001$ & .027 & $.004, .049$ & .011 & .020 \\
-.011 & $-.077, .054$ & .033 & .733 & .008 & $-.027, .042$ & .018 & .656 \\
-.006 & $-.012, .000$ & .003 & .044 & -.002 & $-.005, .000$ & .001 & .066 \\
\hline
\end{tabular}

\section{Sequential Mediation Model Paths:}

a path

b path

c' path

Indirect effect $\left(a^{*} b\right)$

\section{Cross-lag Panel}

Mediation Model Paths:

a path

b path

c' path

Indirect effect $\left(\mathrm{a}^{*} \mathrm{~b}\right)$ 


\section{Fear and Mental Health Symptoms}

In the sequential mediation models (summarized in the bottom-half of Table 1), reappraisal was associated with lower fear, which in turn predicted fewer mental health symptoms (i.e., better mental health). The indirect effect of reappraisal on fewer mental health symptoms, mediated by fear, was statistically significant in both samples.

The cross-lag panel mediation models replicated the sequential mediation models: reappraisal predicted lower fear, which in turn predicted fewer mental health symptoms. The indirect effect of reappraisal on fewer mental health symptoms, mediated by fear, was statistically significant in the weekly model, and marginally significant in the monthly model.

\section{Socially Oriented-Positive Emotions and Health Behaviors}

In the sequential mediation models (summarized in the top-half of Table 2), reappraisal predicted greater socially-oriented positive emotions, which in turn predicted greater health behaviors. The indirect effect of reappraisal on greater health behaviors, mediated by sociallyoriented positive emotions, was statistically significant in both samples.

The cross-lag panel mediation models indicated that reappraisal predicted greater socially-oriented positive emotions, replicating the sequential mediation models. However, unlike the sequential mediation models, socially-oriented positive emotions did not predict greater health behaviors in the cross-lag panel models. Because of this, the indirect effect of reappraisal on health behaviors, mediated by socially-oriented positive emotions, was nonsignificant in the weekly and monthly models ${ }^{1}$.

\footnotetext{
${ }^{1}$ Although these null results should not be interpreted as evidence for the absence of an effect, the $95 \%$ CIs around these null effects are consistent with a negligible negative effect to a small positive effect of socially-oriented positive emotions on health behaviors. Thus, the full range of these $95 \%$ CIs is consistent with the hypothesis that socially-oriented positive emotions are not associated with meaningfully reduced health behaviors.
} 


\section{Socially Oriented-Positive Emotions and Mental Health Symptoms}

In the sequential mediation models (summarized in the bottom-half of Table 2), reappraisal predicted greater socially-oriented positive emotions, which in turn predicted fewer mental health symptoms (i.e., better mental health). The indirect effect of reappraisal on fewer mental health symptoms, mediated by socially-oriented positive emotions, was statistically significant in both samples.

The cross-lag panel mediation models again indicated that reappraisal predicted greater socially-oriented positive emotions, replicating the sequential mediation models. However, unlike the sequential mediation models, socially-oriented positive emotions did not predict mental health symptoms in the cross-lag panel models. Because of this, the indirect effect of reappraisal on mental health symptoms, mediated by socially-oriented positive emotions, was non-significant in the weekly and monthly models. 


\section{Table 2}

Reappraisal Predicting Higher Socially-Oriented Positive Emotions and in Turn Greater Health Behaviors and Better Mental Health (i.e., Fewer Mental Health Symptoms)

\begin{tabular}{|c|c|c|c|c|c|c|c|c|}
\hline \multirow[b]{3}{*}{$\begin{array}{l}\text { Sequential Mediation } \\
\text { Model Paths }\end{array}$} & \multicolumn{8}{|c|}{ Predicting Health Behaviors } \\
\hline & \multicolumn{4}{|c|}{ Sample A $(\mathrm{N}=696)$} & \multicolumn{4}{|c|}{ Sample B $(\mathrm{N}=545)$} \\
\hline & $\mathrm{b}$ & $95 \% \mathrm{CI}$ & $\mathrm{SE}$ & $p$ & $\mathrm{~b}$ & $95 \% \mathrm{CI}$ & $\mathrm{SE}$ & $p$ \\
\hline a path & .314 & $.239, .389$ & .038 & $<.001$ & .392 & $.313, .471$ & .040 & $<.001$ \\
\hline b path & .096 & $.037, .155$ & .030 & .001 & .103 & $.041, .164$ & .031 & .001 \\
\hline$c^{\prime}$ path & -.060 & $-.115,-.004$ & .028 & .035 & -.058 & $-.115,-.001$ & .029 & .048 \\
\hline Indirect effect $(\mathrm{a} * \mathrm{~b}$ & .030 & $.010, .050$ & .010 & .003 & .040 & $.015, .066$ & .013 & .002 \\
\hline \multirow{2}{*}{$\begin{array}{l}\text { Cross-lag Panel } \\
\text { Mediation Model Paths: }\end{array}$} & \multicolumn{4}{|c|}{ Sample A Weekly $(\mathrm{N}=530)$} & \multicolumn{4}{|c|}{ Sample A Monthly $(\mathrm{N}=518)$} \\
\hline & $\mathrm{b}$ & $95 \% \mathrm{CI}$ & $\mathrm{SE}$ & $p$ & $\mathrm{~b}$ & $95 \% \mathrm{CI}$ & $\mathrm{SE}$ & $p$ \\
\hline a path & .159 & $.104, .214$ & .028 & $<.001$ & .117 & $.062, .171$ & .028 & $<.001$ \\
\hline b path & .007 & $-.010, .024$ & .009 & .415 & .007 & $-.016, .031$ & .012 & .529 \\
\hline$c^{\prime}$ path & -.002 & $-.029, .026$ & .014 & .902 & .008 & $-.028, .044$ & .019 & .665 \\
\hline \multirow[t]{2}{*}{ Indirect effect $\left(a^{*} b\right)$} & .001 & $-.002, .004$ & .001 & .420 & .001 & $-.002, .004$ & .001 & .533 \\
\hline & \multicolumn{8}{|c|}{ Predicting Mental Health } \\
\hline \multirow{2}{*}{$\begin{array}{l}\text { Sequential Mediation } \\
\text { Model Paths: }\end{array}$} & \multicolumn{4}{|c|}{ Sample A $(N=696)$} & \multicolumn{4}{|c|}{ Sample B $(N=545)$} \\
\hline & $\mathrm{b}$ & $95 \% \mathrm{CI}$ & $\mathrm{SE}$ & $p$ & $\mathrm{~b}$ & $95 \% \mathrm{CI}$ & $\mathrm{SE}$ & $p$ \\
\hline a path & .316 & $.241, .391$ & .038 & $<.001$ & .391 & $.312, .470$ & .040 & $<.001$ \\
\hline b path & -.155 & $-.223,-.087$ & .034 & $<.001$ & -.194 & $-.257,-.131$ & .032 & $<.001$ \\
\hline$c^{\prime}$ path & -.141 & $-.205,-.076$ & .033 & $<.001$ & -.192 & $-.250,-.134$ & .030 & $<.001$ \\
\hline Indirect effect $(a * b)$ & -.049 & $-.073,-.025$ & .012 & $<.001$ & -.076 & $-.105,-.047$ & .015 & $<.001$ \\
\hline \multirow{2}{*}{$\begin{array}{l}\text { Cross-lag Panel } \\
\text { Mediation Model Paths: }\end{array}$} & \multicolumn{4}{|c|}{ Sample A Weekly $(\mathrm{N}=530)$} & \multicolumn{4}{|c|}{ Sample A Monthly $(\mathrm{N}=518)$} \\
\hline & $\mathrm{b}$ & $95 \% \mathrm{CI}$ & $\mathrm{SE}$ & $p$ & $\mathrm{~b}$ & $95 \% \mathrm{CI}$ & $\mathrm{SE}$ & $p$ \\
\hline a path & .160 & $.105, .215$ & .028 & $<.001$ & .115 & $.061, .169$ & .028 & $<.001$ \\
\hline b path & -.028 & $-.068, .013$ & .021 & .183 & .002 & $-.018, .022$ & .010 & .820 \\
\hline$c^{\prime}$ path & -.028 & $-.094, .037$ & .033 & .400 & .000 & $-.034, .035$ & .018 & .983 \\
\hline Indirect effect $\left(a^{*} b\right)$ & -.004 & $-.011, .002$ & .003 & .195 & .000 & $-.002, .003$ & .001 & .820 \\
\hline
\end{tabular}




\section{Discussion}

Bridging affective science with health psychology, the present research provides several theoretical contributions. When facing a frightening health crisis, it is understandable to want to seek comfort. However, feeling better can come with important costs that can jeopardize not only one's personal health, but also the health of the community. In two diverse samples, across three months of an unfolding pandemic, and using multiple types of longitudinal mediation analyses, we found that successfully reappraising the situation surrounding a pandemic predicted less fear which in turn, predicted better longer-term mental health. However, by reducing fear, successfully reappraising also predicted less engagement in health behaviors that were recommended by the CDC to slow the spread of infection, thereby generating potentially fatal risk to individuals and their communities. This research is among the first to indicate that the very strategies meant to help people cope with the stress of health threats may further jeopardize people's physical health outcomes and provides evidence for the underlying mechanisms of these outcomes. From a public health perspective, our findings suggest that health messages that emphasize reduced fear (e.g., 'keep calm and carry on') may actually backfire and promote fewer health behaviors. These results underscore an unfortunate dilemma of seeking comfort during times of stress, when comfort is most needed.

There is more than one way to seek comfort, however. Moving beyond prior work on the costs of reappraisal (Ford \& Troy, 2019), the present findings suggest a potential solution: reappraisal predicted increased socially-oriented positive emotions (e.g., gratitude, inspiration), even in the face of a global health crisis. Indeed, $89-97 \%$ of our sample reported feeling at least some socially-oriented positive emotions within the first three months of the COVID-19 pandemic (comparable to the prevalence of fear, 80-94\%). Importantly, across all of our well- 
powered longitudinal analyses, we found no evidence that socially-oriented positive emotions interfered with people's motivation to engage in CDC-recommended health behaviors. People were able to experience the comfort of these positive emotional experiences without jeopardizing their own or others' health. In fact, in the sequential mediation models, socially-oriented positive emotions predicted greater engagement in health behaviors. In the cross-lag panel models, the effect of socially-positive emotions of health behaviors was null. Although we caution against interpreting null results as evidence against an effect, the $95 \%$ CIs around these null effects are consistent with a negligible negative effect to a small positive effect.

In addition to these contributions, the present research has several limitations that suggest key directions for future work. First, although the present results replicated across multiple diverse U.S. samples, timeframes, and analysis types, future research is needed to confirm whether reappraisal influences people's responses in the context of other cultures (e.g., which differ in outbreak severity, health behavior guidelines, and culturally-informed responses to emotions) or other types of health threats. Second, we assessed individual's use of reappraisal at a broad level (i.e., changing how one is thinking) and focused on hedonic regulation (i.e., striving to feel better), but reappraisal can be used in many different ways. For example, specific reappraisal tactics may have unique outcomes (e.g., minimizing vs. benefit-finding; Shiota \& Levenson, 2012), reappraisal could be used to increase negative emotions (e.g., to achieve instrumental benefits; Tamir, 2015), or reappraisal could be used to change other people's emotions in the context of a collective crisis (Netzer, Halperin \& Tamir, 2020). Future research would benefit from a more comprehensive examination of reappraisal. Finally, because the present research used observational data, we cannot draw strong causal conclusions. The crosslag panel models provide evidence for directionality, but not necessarily causality. Specifically, by 
including autoregressive paths, these models account for pre-existing individual differences in the predictor variables as well as shared variance with correlated third variables. Despite the strengths of these models, it is possible that third variables change with change in the predictor variables or that third variables predispose individuals to change in the predictor variables. These possibilities are an inherent limitation to non-experimental work and future work is needed to investigate and untangle potential third variables.

This research provides a novel test of the complex trade-offs associated with seeking comfort during a large-scale health threat. Emotion regulation is clearly a powerful tool and as such, must be wielded with caution and knowledge of both its benefits and its costs. 


\section{References}

Aldao, A., Nolen-Hoeksema, S. \& Schweizer, S. (2010). Emotion-regulation strategies across psychopathology: A meta-analytic review. Clinical Psychological Review, 30, 217-237.

Christakis, N. A., \& Fowler, J. H. (2008). The collective dynamics of smoking in a large social network. New England Journal of Medicine, 358(21), 2249-2258

Cook E., \& Chater, A. (2010). Are happier people, healthier people? The relationship between perceived happiness, personal control, BMI and health preventive behaviours. International Journal of Health Promotion and Education, 48(2), 58-64. doi:10.1080/14635240.2010.10708183

Dieng, M., Butow, P. N., Costa, D. S., Morton, R. L., Menzies, S. W., Mireskandari, S., ..., Kasparian, N. A. (2016). Psychoeducational Intervention to Reduce Fear of Cancer Recurrence in People at High Risk of Developing Another Primary Melanoma: Results of a Randomized Controlled Trial. Journal of Clinical Oncology, 34(36), 4405-4414. doi:10.1200/jco.2016.68.2278

Doré, B. P., Tompson, S. H., O'donnell, M. B., An, L. C., Strecher, V., \& Falk, E. B. (2019). Neural Mechanisms of Emotion Regulation Moderate the Predictive Value of Affective and Value-Related Brain Responses to Persuasive Messages. The Journal of Neuroscience, 39(7), 1293-1300. doi:10.1523/jneurosci.1651-18.201

Feigelman, S., Stanton, B., Rubin, J. D., \& Cartelli, N. A. (1993). Effectiveness of family notification efforts and compliance with measles post-exposure prophylaxis. Journal of Community Health, 18(2), 83-93. doi:10.1007/bf01324417 
Feinberg, M., Ford, B. Q., \& Flynn, F. J. (2020). Rethinking reappraisal: The double-edged sword of regulating negative emotions in the workplace. Organizational Behavior and Human Decision Processes, 161, 1-19. doi:10.1016/j.obhdp.2020.03.005

Folkman, S. (1997). Positive psychological states and coping with severe stress. Social Science \& Medicine, 45(8), 1207-1221. doi:10.1016/s0277-9536(97)00040-3

Folkman, S., \& Greer, S. (2000). Promoting psychological well-being in the face of serious illness: When theory, research and practice inform each other. Psycho-Oncology, 9(1), 11-19. doi:10.1002/(sici)1099-1611(200001/02)9:13.0.co;2-z

Frijda, N. (1986). The emotions. London: Cambridge University Press

Ford, B. Q., Feinberg, M., Lam, P., Mauss, I. B., \& John, O. P. (2019). Using reappraisal to regulate negative emotion after the 2016 U.S. Presidential election: Does emotion regulation trump political action? Journal of Personality and Social Psychology, 117(5), 998-1015. doi:10.1037/pspp0000200

Ford, B. Q., Karnilowicz, H. R., \& Mauss, I. B. (2017). Understanding reappraisal as a multicomponent process: The psychological health benefits of attempting to use reappraisal depend on reappraisal success. Emotion, 17(6), 905-911. doi: $10.1037 / \mathrm{emo} 0000310$

Ford, B. Q., \& Troy, A. S. (2019). Reappraisal reconsidered: A closer look at the costs of an acclaimed emotion-regulation strategy. Current Directions in Psychological Science, 28, 195-203. doi:10.1177/0963721419827526

Gross, J. J. Emotion regulation: Current status and future prospects. Psychological Inquiry, 26, $1-26(2015)$. 
Harper, C. A., Satchell, L. P., Fido, D., \& Latzman, R. D. (2020). Functional fear predicts public health compliance in the COVID-19 pandemic. International Journal of Mental Health and Addiction.

Heiy, J. E. \& Cheavens, J. S. Back to basics: A naturalistic assessment of the experience and regulation of emotion. Emotion 14, 878-891 (2014).

Jans-Beken L., Jacobs, N., Janssens, M., Peeters, S., Reijnders, J., Lechner, L., \& Lataster, J. (2019). Gratitude and health: An updated review. The Journal of Positive Psychology, 140. doi:10.1080/17439760.2019.1651888

Kline, R. B. (2015). Principles and practice of structural equation modeling. Guilford Publications.

Kotov, R., Gamez, W., Schmidt, F., \& Watson, D. (2010). Linking “big” personality traits to anxiety, depressive, and substance use disorders: A meta-analysis. Psychological Bulletin, 136(5), 768-821. https://doi.org/10.1037/a0020327

Kraaij, V., Garnefski, N., Schroevers, M. J., Weijmer, J., \&; Helmerhorst, F. (2010). Cognitive coping, Goal adjustment, and depressive and anxiety symptoms in people undergoing infertility treatment. Journal of Health Psychology, 15(6), 876-886. doi:10.1177/1359105309357251

Kraaij, V., Van Der Veek, S. M. C., Garnefski, N., Schroevers, M., Witlox, R., \& Maes, S. (2008). Coping, goal adjustment, and psychological well-being in hiv-infected men who have sex with men. AIDS Patient Care and STDs, 22(5), 395-402. doi:10.1089/apc.2007.0145

Maxwell, S. E., \& Cole, D. A. (2007). Bias in cross-sectional analyses of longitudinal mediation. Psychological Methods, 12(1), 23. 
Mayne, T. J. (1999). Negative affect and health: The importance of being earnest. Cognition \& Emotion, 13(5), 601-635. doi:10.1080/026999399379203

Mennin, D. S. (2005). Emotion and the Acceptance-Based Approaches to the Anxiety Disorders. In S. M. Orsillo \& L. Roemer (Eds.), Series in anxiety and related disorders. Acceptance and mindfulness-based approaches to anxiety: Conceptualization and treatment (p. 3768). Springer Science + Business Media. https://doi.org/10.1007/0-387-25989-9_2

Moskowitz, J. T., Folkman, S., Collette, L., \& Vittinghoff, E. (1996). Coping and mood during aids-related caregiving and bereavement. Annals of Behavioral Medicine, 18(1), 49-57. doi:10.1007/bf02903939

Netzer, L., Halperin, E., \& Tamir, M. (2020). Be Afraid, Be Very Afraid! Motivated Intergroup Emotion Regulation. Personality and Social Psychology Bulletin, 46(11), 1596-1613. https://doi.org/10.1177/0146167220910833

Peh, C. X., Liu, J., Bishop, G. D., Chan, H. Y., Chua, S. M., Kua, E. H., \& Mahendran, R. (2016). Emotion regulation and emotional distress: The mediating role of hope on reappraisal and anxiety/depression in newly diagnosed cancer patients. Psycho-Oncology, 26(8), 1191-1197. doi:10.1002/pon.4297

Preacher, K. J., Zhang, Z., \& Zyphur, M. J. (2011). Alternative methods for assessing mediation in multilevel data: The advantages of multilevel SEM. Structural Equation Modeling, $18(2), 161-182$.

Radloff, L.S. (1977). The CED-D scale: A self-report depression scale for research in the general population. Applied Psychological Measurement, 1, 385-401. 
Russell, J. A., \& Barrett, L. F. (1999). Core affect, prototypical emotional episodes, and other things called emotion: Dissecting the elephant. Journal of Personality and Social Psychology, 76(5), 805-819. doi:10.1037/0022-3514.76.5.805

Selig, J. P., \& Preacher, K. J. (2009). Mediation models for longitudinal data in developmental research. Research in Human Development, 6(2-3), 144-164.

Shepherd, L., \& Smith, M. A. (2017). The role of fear in predicting sexually transmitted infection screening. Psychology \& Health, 32(7), 876-894. doi:10.1080/08870446.2017.1307375

Shiota, M. N., \& Levenson, R. W. (2012). Turn down the volume or change the channel? Emotional effects of detached versus positive reappraisal. Journal of Personality and Social Psychology, 103(3), 416-429. doi:10.1037/a0029208

Snaith, R. P. (2003). The hospital anxiety and depression scale. Health and Quality of Life Outcomes, 1(1), 1-4.

Tamir, M. (2015). Why Do People Regulate Their Emotions? A Taxonomy of Motives in Emotion Regulation. Personality and Social Psychology Review, 20(3), 199-222. https://doi.org/10.1177/1088868315586325

Tesson, Richards, I., Porter, D., Phillips, K., Rankin, N., Costa, D., . . Butow, P. (2017). Women's preferences for contralateral prophylactic mastectomy following unilateral breast cancer: What risk-reduction makes it worthwhile? The Breast, 31, 233-240. doi:10.1016/j.breast.2016.11.025

Troy, A. S., Shallcross, A. J., \& Mauss, I. B. (2013). A person-by-situation approach to emotion regulation: Cognitive reappraisal can either help or hurt, depending on the context. Psychological Science, 24, 2505-2514. doi:10.1177/0956797 615627417 
Uusberg, A., Taxer, J. L., Yih, J., Uusberg, H., \& Gross, J. J. (2019). Reappraising reappraisal. Emotion Review, 11, 267-282. https://doi.org/10.1177/1754073919862617

van't Wout, M., Chang, L. J., \& Sanfey, A. G. (2010). The influence of emotion regulation on social interactive decision-making. Emotion, 10, 815-821. doi:10.1037\%2Fa0020069

Wang, Yi, J., He, J., Chen, G., Li, L., Yang, Y., \& Zhu, X. (2013). Cognitive emotion regulation strategies as predictors of depressive symptoms in women newly diagnosed with breast cancer. Psycho-Oncology, 23(1), 93-99. doi:10.1002/pon.3376 


\section{Supplementary Online Material}

\section{Data Quality Checks}

To improve data quality for both samples, each time point excluded participants who failed to pass all attention checks, provided incomplete data, or took the survey more than once. A table that lists all data exclusions at each time point for each sample, with additional information of why each exclusion occurred is available at: https://osf.io/y8kp3/?view_only=32bd0b2dc3a842a38e90218b031852a7.

In addition, for Sample A, T1 included a screener item that appeared at the start of the survey, where participants needed to indicate that they had heard of COVID-19 to be eligible for the study.

\section{Materials: Reappraisal Items}

Participants completed separate ratings for how frequently they used reappraisal and how successfully they used reappraisal when feeling stressed about the COVID-19 outbreak at T1, T1b, T2, T2b, and T3 in Sample A and at T2, T2b, and T3 in Sample B. Participants rated four reappraisal frequency items on a scale from 0 (I never try this) to 6 (I always try this), with items: "When you want to feel less negative emotion about the recent coronavirus outbreak (such as anxiety, disgust, or frustration), do you try to change the way you're thinking about the outbreak?"; "When you are feeling stressed about the outbreak, do you try to make yourself think about the outbreak in a way that helps you stay calm?"; "When you want to feel more positive emotion about the outbreak (such as hope or relief), do you try to change the way you're thinking about the outbreak?"; and "Do you try to control your emotions about the outbreak by changing the way you think about the outbreak?" 
Participants rated their reappraisal success on a scale from 0 (I am definitely unable to do this) to 6 (I am definitely able to do this), with items: "When you want to feel less negative emotion about the recent outbreak (such as anxiety, disgust, or frustration), are you able to change the way you're thinking about the outbreak, if you try?"; "When you are feeling stressed about the outbreak, are you successful at making yourself think about the outbreak in a way that helps you stay calm, if you try?"; "When you want to feel more positive emotion about the outbreak (such as hope or relief), are you successful at changing the way you're thinking about the outbreak, if you try?"; and "If you try, are you successful at controlling your emotions about the outbreak by changing the way you think about the outbreak?"

\section{Materials: Health Behavior Items}

In the weekly assessments, beginning with T2a, participants rated how often they engaged in the following actions over the past week: "practiced strict hygiene (e.g., washing hands, disinfecting surfaces, not touching your face)," "wore a face mask," "stayed at least 2 arms lengths (approximately 6 feet) away from other people, except for the people you live with," and "engaged in self-isolation (e.g., avoided leaving your home)."

In the monthly assessments, beginning with T2, participants rated how often they engaged in the following actions over the past month: "wore a face mask," "sanitized surfaces in your house after touching," "took preventative measures for myself (e.g., increased hand washing, used hand sanitizer)," "stayed at least 2 arms lengths (approximately 6 feet) away from other people, except for the people I live with," and "engaged in self-isolation (e.g., avoided leaving your home for any reason)." 


\section{Social Negative and Non-social Positive Emotions}

In addition to the analyses that focused on fear and socially-oriented positive emotions (reported in the main text), we also tested indirect pathways through social negative emotions (see Supplementary Table 2) and non-social positive emotions (see Supplementary Table 3). To assess social negative emotions, we used the following triplets: "ashamed, humiliated, embarrassed" and "guilty, repentant, blameworthy" (alpha $=.72-.83)$. To assess non-social positive emotions, we used the following triplet and couplet: "glad, happy, joyful" and "amused, entertained" (alpha $=.71-.80)$. These results indicate that the pattern of results reported in the primary text are specific to fear and socially-oriented positive emotions.

\section{Intended Future Health Behaviors}

In addition to past health behaviors (reported in the main text), we also tested effects on intended future health behaviors. In the monthly assessments (beginning with T2), we assessed participants' intention to engage in the same COVID-19 preventative health behaviors described in the main manuscript over the next four weeks. Response options ranged from 0 (I will definitely not do this) to 4 (I will definitely do this). We computed a mean health behaviors composite at each monthly time point $($ alpha $=.75-.82)$. Intended health behaviors were not assessed in the weekly assessments. Tables with results for both the sequential mediation model and the cross-lag panel model for the monthly assessments are available at https://osf.io/y8kp3/?view_only=32bd0b2dc3a842a38e90218b031852a7. These results suggest that the pattern of results reported in the primary text are largely mirrored when considering the health behaviors that people intend to engage in. 


\section{Well-being}

In addition to mental health symptoms (reported in the main text), we also tested effects on well-being. We assessed two aspects of well-being: psychological well-being and life satisfaction. In the monthly assessments, we used the Meaning in Life Questionnaire (Steger, Frazier, Oishi, \& Kaler, 2006) to assess psychological well-being over the past four weeks and the Satisfaction with Life Scale (Diener, Emmons, Larsen, \& Griffin, 1986) to assess life satisfaction over the past four weeks. First, we z-standardized each scale score at each timepoint. Second, we computed a mean well-being health composite of the two z-scored variables at each timepoint (alpha $=.77-.81)$. In the weekly assessments, we used single item measures to assess psychological well-being and life satisfaction in the past week: "This week, I felt like my life had a clear sense of meaning and purpose." and "I felt satisfied with life this week." Response options ranged from 0 (I do not agree with this at all) to 6 (I completely agree with this). We computed the mean of the two items at each timepoint (alpha $=.79-.82)$. Tables with results for both the sequential mediation model and the cross-lag panel mediation model are available at https://osf.io/y8kp3/?view_only=32bd0b2dc3a842a38e90218b031852a7. These results suggest that the pattern of results reported in the primary text are largely mirrored when considering measures of well-being. 


\section{Supplementary Table 1}

Means and Standard Deviations of Study Variables

\begin{tabular}{|c|c|c|c|c|c|c|c|c|c|}
\hline Sample A & $\mathbf{T} 1$ & T1b & T1c & $\mathbf{T} 2$ & T2a & $\mathbf{T} 2 \mathrm{~b}$ & T2c & T3 & $\mathbf{T 4}$ \\
\hline Reappraisal & $3.76(1.39)$ & $3.47(1.39)$ & -- & $3.54(1.45)$ & -- & $3.52(1.42)$ & -- & $3.57(1.48)$ & -- \\
\hline Fear & $\mathrm{X}$ & $3.00(1.68)$ & $2.95(1.71)$ & $2.71(1.81)$ & $2.44(1.69)$ & $2.14(1.71)$ & $2.11(1.70)$ & $1.95(1.75)$ & $\mathrm{X}$ \\
\hline Social Positive Emotions & -- & $1.97(1.55)$ & $2.15(1.47)$ & $2.54(1.65)$ & $2.60(1.61)$ & $2.66(1.65)$ & $2.64(1.65)$ & $2.77(1.64)$ & $\mathrm{X}$ \\
\hline Social Negative Emotions & $\mathrm{X}$ & $0.78(1.22)$ & $0.82(1.22)$ & $0.79(1.23)$ & $0.66(1.13)$ & $0.59(1.07)$ & $0.68(1.13)$ & $0.61(1.12)$ & $\mathrm{X}$ \\
\hline Non-Social Positive Emotions & $\mathrm{X}$ & $1.18(1.36)$ & $1.31(1.39)$ & $1.60(1.45)$ & $1.68(1.51)$ & $1.91(1.56)$ & $1.87(1.61)$ & $1.91(1.57)$ & $\mathrm{X}$ \\
\hline Past Health Behaviors & $\mathrm{X}$ & -- & -- & $2.64(0.76)$ & $3.97(0.72)$ & $3.07(0.70)$ & $3.19(0.74)$ & $3.07(0.83)$ & $3.12(0.80)$ \\
\hline Future Health Behaviors & $\mathrm{X}$ & -- & -- & $2.97(0.79)$ & -- & -- & -- & $3.20(0.85)$ & $3.20(0.83)$ \\
\hline Mental Health Symptoms & $\mathrm{X}$ & -- & -- & $0.00(0.96)$ & $2.33(1.63)$ & $2.28(1.73)$ & $2.06(1.66)$ & $0.00(0.96)$ & $0.00(0.96)$ \\
\hline Well-being & $\mathrm{X}$ & -- & -- & $0.00(0.90)$ & $2.80(1.62)$ & $2.95(1.62)$ & $2.99(1.59)$ & $0.00(0.91)$ & $0.00(0.92)$ \\
\hline Sample B & T1* & T1b & T1c & $\mathbf{T} 2$ & T2a & $\mathbf{T} 2 \mathrm{~b}$ & T2c & $\mathbf{T 3}$ & $\mathbf{T 4}$ \\
\hline Reappraisal & -- & -- & -- & $3.37(1.48)$ & -- & $\mathrm{X}$ & -- & $\mathrm{X}$ & -- \\
\hline Fear & -- & -- & -- & $\mathrm{x}$ & $2.32(1.64)$ & $1.96(1.59)$ & $1.85(1.56)$ & $1.70(1.63)$ & $\mathrm{X}$ \\
\hline Social Positive Emotions & -- & -- & -- & $\mathrm{X}$ & $2.89(1.66)$ & $2.99(1.57)$ & $2.89(1.62)$ & $3.03(1.67)$ & $\mathrm{X}$ \\
\hline Social Negative Emotions & -- & -- & -- & $\mathrm{x}$ & $0.58(0.98)$ & $0.49(0.91)$ & $0.49(0.97)$ & $0.45(0.92)$ & $\mathrm{X}$ \\
\hline Non-Social Positive Emotions & -- & -- & -- & $\mathrm{X}$ & $1.96(1.57)$ & $2.12(1.51)$ & $2.08(1.60)$ & $2.18(1.61)$ & $\mathrm{X}$ \\
\hline Past Health Behaviors & -- & -- & -- & $\mathrm{x}$ & $\mathrm{X}$ & $\mathrm{x}$ & $\mathrm{x}$ & $\mathrm{x}$ & $2.94(0.84)$ \\
\hline Future Health Behaviors & -- & -- & -- & $\mathrm{X}$ & -- & -- & -- & $3.03(0.89)$ & $\mathrm{X}$ \\
\hline Mental Health Symptoms & $\mathrm{X}$ & -- & -- & $\mathrm{X}$ & $\mathrm{X}$ & $\mathrm{X}$ & $\mathrm{X}$ & $\mathrm{X}$ & $0.00(0.96)$ \\
\hline Well-being & $\mathrm{X}$ & -- & -- & $\mathrm{X}$ & $\mathrm{X}$ & $\mathrm{X}$ & $\mathrm{X}$ & $\mathrm{X}$ & $0.00(0.92)$ \\
\hline
\end{tabular}

Note. Mean $(S D)$ are shown. A dash indicates that a variable was not assessed. An ' $\mathrm{X}$ ' indicates that a variable was assessed but was not included in the present analyses. All variables are on a 0-6 scale, with the exception of Mental Health and Wellbeing at T2, T3, and T4, which are z-scores. *Sample B completed a T1 survey in mid-February which included measures of mental health and well-being, but it did not include any measures related to COVID (e.g., emotion regulation or emotional experiences in response to COVID, health behaviors), and as such, is not included in any analyses reported in the present paper. 


\section{Supplementary Table 2}

Reappraisal Predicting Health Behaviors and Mental Health Symptoms via Social Negative Emotions

\section{Predicting Health Behaviors}

Sequential

Mediation Model

Paths

a path

b path

c' path

Indirect effect $(a * b$

Cross-lag Panel

Mediation Model

Paths:

a path

b path

c' path

Indirect effect $(a * b)$

\begin{tabular}{cccccccc}
\hline \multicolumn{3}{c}{ Sample A (N=696) } & \multicolumn{5}{c}{ Sample B (N=545) } \\
\hline $\mathrm{b}$ & $95 \% \mathrm{CI}$ & $\mathrm{SE}$ & $p$ & $\mathrm{~b}$ & $95 \% \mathrm{CI}$ & $\mathrm{SE}$ & $p$ \\
\hline & & & & & & \\
\hline .006 & $-.054, .067$ & .031 & .837 & -0.087 & $-.137,-.037$ & .026 & .001 \\
-.066 & $-.145, .013$ & .040 & .104 & 0.032 & $-.072, .136$ & .053 & .548 \\
-.028 & $-.080, .025$ & .027 & .300 & -0.013 & $-.067, .040$ & .027 & .631 \\
.000 & $-.004, .004$ & .002 & .839 & -0.003 & $-.012, .006$ & .005 & .555 \\
\hline
\end{tabular}

Sample A Weekly $(\mathrm{N}=530)$

Sample A Monthly $(\mathrm{N}=518)$

\begin{tabular}{cccccccc}
\hline $\mathrm{b}$ & $95 \% \mathrm{CI}$ & $\mathrm{SE}$ & $p$ & $\mathrm{~b}$ & $95 \% \mathrm{CI}$ & $\mathrm{SE}$ & $p$ \\
\hline-.017 & $-.053, .019$ & .018 & .353 & -.002 & $-.041, .037$ & .020 & .910 \\
.004 & $-.020, .027$ & .012 & .758 & -.028 & $-.059, .002$ & .016 & .070 \\
.000 & $-.027, .028$ & .014 & .987 & .007 & $-.029, .043$ & .018 & .718 \\
.000 & $.000, .000$ & .000 & .770 & .000 & $-.001, .001$ & .001 & .910 \\
\hline
\end{tabular}

Sample A $(\mathrm{N}=696) \quad$ Predicting Mental Health

Sequential

Mediation Model

Paths:

a path

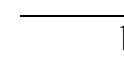

b $\quad 95 \% \mathrm{CI}$

Sample B (N=545)

b path

.004

c' path

Indirect effect $\left(a^{*} b\right)$

Cross-lag Panel

Mediation Model

Paths:

a path

b path

.534

$-.178$

$\mathrm{SE}$

$p \quad \mathrm{~b}$

b

$95 \% \mathrm{CI}$

$\mathrm{SE} \quad p$

$.004 \quad-.057, .065 \quad .031$

$031 \quad .899$

$-.086$

$-.136,-.036 \quad .026 \quad .001$

.002

$.455, .613$

$.040<.001$

.587

$.492, .681$

$.048<.001$

$-.230,-.125 \quad .027<.001$

$-.216$

$-.264,-.168$

$.024<.001$

$-.030, .035$

$.017 \quad .899$

$-.051$

$-.081,-.020$

$.016 \quad .001$

Sample A Weekly $(\mathrm{N}=530)$

Sample A Monthly (N=518)

\begin{tabular}{cccccccc}
\hline $\mathrm{b}$ & $95 \% \mathrm{CI}$ & $\mathrm{SE}$ & $p$ & $\mathrm{~b}$ & $95 \% \mathrm{CI}$ & $\mathrm{SE}$ & $p$ \\
\hline-.017 & $-.053, .019$ & .018 & .360 & -.001 & $-.040, .038$ & .020 & .949 \\
.056 & $-.003, .114$ & .030 & .062 & .057 & $.029, .084$ & .014 & $<.001$ \\
-.039 & $-.104, .025$ & .033 & .234 & .001 & $-.033, .035$ & .017 & .944 \\
-.001 & $-.003, .001$ & .001 & .411 & .000 & $-.002, .002$ & .001 & .949 \\
\hline
\end{tabular}




\section{Supplementary Table 3}

Reappraisal Predicting Health Behaviors and Mental Health Symptoms via Non-social Positive Emotions

\section{Predicting Health Behaviors}

\section{Sequential Mediation Model Paths}

\begin{tabular}{cccccccc}
\hline \multicolumn{3}{c}{ Sample A $(\mathrm{N}=696)$} & & \multicolumn{5}{c}{ Sample B (N=545) } \\
\hline $\mathrm{b}$ & $95 \% \mathrm{CI}$ & $\mathrm{SE}$ & $p$ & $\mathrm{~b}$ & $95 \% \mathrm{CI}$ & $\mathrm{SE}$ & $p$ \\
\hline .315 & $.248, .382$ & .034 & $<.001$ & .364 & $.291, .437$ & .037 & $<.001$ \\
-.002 & $-.073, .069$ & .036 & .958 & .059 & $-.013, .130$ & .036 & .108 \\
-.024 & $-.082, .034$ & .030 & .417 & -.040 & $-.099, .018$ & .030 & .173 \\
-.001 & $-.023, .022$ & .011 & .958 & .021 & $-.005, .048$ & .013 & .112 \\
\hline & Sample A Weekly (N=530) & & Sample A Monthly (N=518) \\
\hline $\mathrm{b}$ & $95 \% \mathrm{CI}$ & $\mathrm{SE}$ & $p$ & $\mathrm{~b}$ & $95 \% \mathrm{CI}$ & $\mathrm{SE}$ & $p$ \\
\hline .181 & $.127, .235$ & .027 & $<.001$ & .189 & $.136,242$ & .027 & $<.001$ \\
-.001 & $-.021, .019$ & .010 & .955 & -.011 & $-.038, .016$ & .014 & .435 \\
.004 & $-.023, .032$ & .014 & .763 & .011 & $-.025, .048$ & .019 & .539 \\
.000 & $-.004, .004$ & .002 & .955 & -.002 & $-.007, .003$ & .003 & .438 \\
\hline
\end{tabular}

a path

\section{Predicting Mental Health}

\section{Cross-lag Panel}

Mediation Model Paths:

a path

b path

c' path

Indirect effect $\left(a^{*} b\right)$

\begin{tabular}{cccccccc}
\hline \multicolumn{3}{c}{ Sample A (N=696) } & & \multicolumn{5}{c}{ Sample B (N=545) } \\
\hline $\mathrm{b}$ & $95 \%$ CI & $\mathrm{SE}$ & $p$ & $\mathrm{~b}$ & $95 \%$ CI & $\mathrm{SE}$ & $p$ \\
\hline .316 & $.249, .383$ & .034 & $<.001$ & .363 & $.290, .436$ & .037 & $<.001$ \\
-.168 & $-.250,-.086$ & .042 & $<.001$ & -.266 & $-.337,-.194$ & .036 & $<.001$ \\
-.132 & $-.199,-.064$ & .034 & $<.001$ & -.175 & $-.232,-.118$ & .029 & $<.001$ \\
-.053 & $-.081,-.025$ & .014 & $<.001$ & -.096 & $-.129,-.064$ & .016 & $<.001$ \\
\hline & Sample A Weekly (N=530) & & & Sample A Monthly $(\mathrm{N}=518)$ \\
\hline $\mathrm{b}$ & $95 \%$ CI & $\mathrm{SE}$ & $p$ & $\mathrm{~b}$ & $95 \% \mathrm{CI}$ & $\mathrm{SE}$ & $p$ \\
\hline .182 & $.128, .236$ & .028 & $<.001$ & .188 & $.135, .241$ & .027 & $<.001$ \\
-.023 & $-.070, .025$ & .024 & .346 & .022 & $-.002, .045$ & .012 & .069 \\
-.029 & $-.095, .037$ & .034 & .388 & .000 & $-.034, .034$ & .018 & 1.000 \\
-.004 & $-.013, .005$ & .004 & .351 & .004 & $.000, .009$ & .002 & .078 \\
\hline
\end{tabular}

\section{Sequential Mediation \\ Model Paths:}

a path

b path

c' path

Indirect effect $(a * b)$

\section{Cross-lag Panel}

Mediation Model Paths:

a path

b path

c' path

Indirect effect $\left(a^{*} \mathrm{~b}\right)$ 


\section{OSF Supplementary Online Material}

\section{Data Quality Checks}

All data points included one or two attention checks: the first was embedded among other items and asked participants to select a specific option. The second was at the end of the survey and asked participants to select the option that best summarized the topic of the survey. In the tables below, the column labelled 'Attention Check Failures' is the number of participants who did not pass either of these checks. Participants with incomplete data were automatically excluded because they did not complete the attention checks ('Survey Not Completed' column). The final step was checking that all participants had unique IDs. If there were any duplicate IDs and both were complete entries, the second entry was excluded ('Repeat IDs' column).

Supplementary Table 4.

Attention Check Details for Sample A

\begin{tabular}{lccccc}
\hline & Initial N & $\begin{array}{c}\text { Survey Not } \\
\text { Completed }\end{array}$ & $\begin{array}{c}\text { Attention } \\
\text { Check Failures }\end{array}$ & Repeat IDs & Final N \\
\hline Time 1 & 940 & 91 & 43 & 0 & 724 \\
Time 1a & 510 & 0 & - & - & 510 \\
Time 1b & 634 & 8 & 17 & 3 & 606 \\
Time 1c & 647 & 19 & 7 & 2 & 619 \\
Time 2 & 630 & 33 & 15 & 1 & 581 \\
Time 2a & 609 & 12 & 8 & 1 & 588 \\
Time 2b & 635 & - & - & 15 & 483 \\
Time 2c & 602 & 19 & 4 & 1 & 578 \\
Time 3 & 599 & 40 & 7 & 0 & 552 \\
Time 4 & 519 & 30 & 6 & 0 & 483 \\
\hline
\end{tabular}

Supplementary Table 5.

Attention Check Details for Sample B

\begin{tabular}{lccccc}
\hline & Initial N & $\begin{array}{c}\text { Survey Not } \\
\text { Completed }\end{array}$ & $\begin{array}{c}\text { Attention } \\
\text { Check Failures }\end{array}$ & Repeat IDs & Final N \\
\hline Time 1 & 966 & 101 & 19 & 5 & 841 \\
Time 2 & 604 & 58 & 13 & 0 & 533 \\
Time 2a & 507 & 6 & 6 & 0 & 495 \\
Time 2b & 511 & 15 & 12 & 0 & 484 \\
Time 2c & 515 & 15 & 1 & 0 & 499 \\
Time 3 & 519 & 29 & 5 & 0 & 485 \\
Time 4 & 483 & 25 & 2 & 1 & 455 \\
\hline
\end{tabular}




\section{Intended Future Health Behaviors}

In addition to past health behaviors (reported in the main text), we also tested effects on intended future health behaviors. In the monthly assessments (beginning with T2), we assessed participants' intention to engage in the same COVID-19 preventative health behaviors described in the main manuscript over the next four weeks. Response options ranged from 0 (I will definitely not do this) to 4 (I will definitely do this). We computed a mean health behaviors composite at each monthly time point $($ alpha $=.75-.82)$. Intended health behaviors were not assessed in the weekly assessments. See the top-half of Supplementary Table 6 for the role of fear in predicting future health behaviors and see the top-half of Supplementary Table 7 for the role of socially-oriented positive emotions in predicting future health behaviors. These results suggest that the pattern of results reported in the primary text are largely mirrored when considering the health behaviors that people intend to engage in.

\section{Well-being}

In addition to mental health symptoms (reported in the main text), we also tested effects on well-being. We assessed two aspects of well-being: psychological well-being and life satisfaction. In the monthly assessments, we used the Meaning in Life Questionnaire (Steger, Frazier, Oishi, \& Kaler, 2006) to assess psychological well-being over the past four weeks and the Satisfaction with Life Scale (Diener, Emmons, Larsen, \& Griffin, 1986) to assess life satisfaction over the past four weeks. First, we z-standardized each scale score at each timepoint. Second, we computed a mean well-being health composite of the two z-scored variables at each timepoint (alpha $=.77-.81)$. In the weekly assessments, we used single item measures to assess psychological well-being and life satisfaction in the past week: "This week, I felt like my life had a clear sense of meaning and purpose." and "I felt satisfied with life this week." Response 
options ranged from 0 (I do not agree with this at all) to 6 (I completely agree with this). We computed the mean of the two items at each timepoint (alpha $=.79-.82$ ). See the bottom-half of Supplementary Table 6 for the role of fear in predicting well-being and see the bottom-half of Supplementary Table 7 for the role of socially-oriented positive emotions in predicting wellbeing. These results suggest that the pattern of results reported in the primary text are largely mirrored when considering measures of well-being. 


\section{Supplementary Table 6}

Reappraisal Predicting Future Health Behaviors and Well-being Via Fear

\begin{tabular}{|c|c|c|c|c|c|c|c|c|}
\hline \multirow{3}{*}{$\begin{array}{l}\text { Sequential Mediation } \\
\text { Model Paths }\end{array}$} & \multicolumn{8}{|c|}{ Predicting Future Health Behaviors } \\
\hline & \multicolumn{4}{|c|}{ Sample A $(N=696)$} & \multicolumn{4}{|c|}{ Sample B $(N=545)$} \\
\hline & $\mathrm{b}$ & $95 \% \mathrm{CI}$ & $\mathrm{SE}$ & $p$ & $\mathrm{~b}$ & $95 \% \mathrm{CI}$ & $\mathrm{SE}$ & $p$ \\
\hline a path & -.310 & $-.396,-.225$ & .044 & $<.001$ & -.479 & $-.554,-.403$ & .038 & $<.001$ \\
\hline b path & .149 & $.098, .200$ & .026 & $<.001$ & .214 & $.148, .279$ & .034 & $<.001$ \\
\hline$c^{\prime}$ path & .059 & $.006, .112$ & .027 & .028 & .066 & $.004, .127$ & .031 & .036 \\
\hline Indirect effect $(\mathrm{a} * \mathrm{~b}$ & -.046 & $-.067,-.026$ & .010 & $<.001$ & -.102 & $-.138,-.067$ & .018 & $<.001$ \\
\hline \multirow[b]{2}{*}{$\begin{array}{l}\text { Cross-lag Panel } \\
\text { Mediation Model Paths*: }\end{array}$} & & & & & \multicolumn{4}{|c|}{ Sample A Monthly $(\mathrm{N}=518)$} \\
\hline & & & & & $\mathrm{b}$ & $95 \% \mathrm{CI}$ & $\mathrm{SE}$ & $p$ \\
\hline a path & & & & & -.088 & $-.145,-.030$ & .029 & .003 \\
\hline b path & & & & & .020 & $-.003, .044$ & .012 & .092 \\
\hline$c^{\prime}$ path & & & & & .006 & $-.035, .047$ & .021 & .782 \\
\hline \multirow[t]{3}{*}{ Indirect effect $(a * b)$} & & & & & -.002 & $-.004, .001$ & .001 & .142 \\
\hline & \multicolumn{8}{|c|}{ Predicting Well-being } \\
\hline & \multicolumn{4}{|c|}{ Sample A $(N=696)$} & \multicolumn{4}{|c|}{ Sample B $(\mathrm{N}=545)$} \\
\hline $\begin{array}{l}\text { Sequential Mediation } \\
\text { Model Paths: }\end{array}$ & $\mathrm{b}$ & $95 \% \mathrm{CI}$ & $\mathrm{SE}$ & $p$ & $\mathrm{~b}$ & $95 \% \mathrm{CI}$ & $\mathrm{SE}$ & $p$ \\
\hline a path & -.309 & $-.395,-.224$ & .044 & $<.001$ & -.479 & $-.554,-.403$ & .038 & $<.001$ \\
\hline b path & -.142 & $-.200,-.084$ & .030 & $<.001$ & -.205 & $-.271,-.139$ & .034 & $<.001$ \\
\hline$c^{\prime}$ path & .123 & $.062, .184$ & .031 & $<.001$ & .111 & $.050, .172$ & .031 & $<.001$ \\
\hline Indirect effect $\left(a^{*} b\right)$ & .044 & $.022, .066$ & .011 & $<.001$ & .098 & $.063, .133$ & .018 & $<.001$ \\
\hline & \multicolumn{4}{|c|}{ Sample A Weekly $(\mathrm{N}=530)$} & \multicolumn{4}{|c|}{ Sample A Monthly $(\mathrm{N}=518)$} \\
\hline Mediation Model Paths: & $\mathrm{b}$ & $95 \% \mathrm{CI}$ & $\mathrm{SE}$ & $p$ & $\mathrm{~b}$ & $95 \% \mathrm{CI}$ & $\mathrm{SE}$ & $p$ \\
\hline a path & -.066 & $-.122,-.010$ & .029 & .022 & -.087 & $-.144,-.030$ & .029 & .003 \\
\hline b path & -.054 & $-.091,-.016$ & .019 & .005 & -.007 & $-.023, .009$ & .008 & .387 \\
\hline$c^{\prime}$ path & .057 & $-.004, .118$ & .031 & .065 & .021 & $-.009, .051$ & .015 & .164 \\
\hline Indirect effect $\left(a^{*} b\right)$ & .004 & $.000, .007$ & .002 & .073 & .001 & $-.001, .002$ & .001 & .406 \\
\hline
\end{tabular}

Note. *Future health behaviors were not assessed in the weekly assessments. 


\section{Supplementary Table 7}

Reappraisal Predicting Future Health Behaviors and Well-being Via Social Positive Emotions

\begin{tabular}{|c|c|c|c|c|c|c|c|c|}
\hline \multirow{3}{*}{$\begin{array}{l}\text { Sequential Mediation } \\
\text { Model Paths }\end{array}$} & \multicolumn{8}{|c|}{ Predicting Future Health Behaviors } \\
\hline & \multicolumn{4}{|c|}{ Sample A $(\mathrm{N}=696)$} & \multicolumn{4}{|c|}{ Sample B $(\mathrm{N}=545)$} \\
\hline & $\mathrm{b}$ & $95 \% \mathrm{CI}$ & SE & $p$ & $\mathrm{~b}$ & $95 \% \mathrm{CI}$ & SE & $p$ \\
\hline a path & .313 & $.238, .389$ & .038 & $<.001$ & .392 & $.313, .471$ & .040 & $<.001$ \\
\hline b path & .119 & $.061, .177$ & .030 & $<.001$ & .094 & $.030, .158$ & .033 & .004 \\
\hline c' path & -.028 & $-.082, .027$ & .028 & .318 & -.077 & $-.137,-.017$ & .031 & .012 \\
\hline \multirow[t]{2}{*}{ Indirect effect $\left(a^{*} b\right)$} & .037 & $.017, .058$ & .010 & $<.001$ & .037 & $.011, .063$ & .013 & .006 \\
\hline & & & & & \multicolumn{4}{|c|}{ Sample A Monthly $(\mathrm{N}=518)$} \\
\hline Mediation Model Paths*: & & & & & $\mathrm{b}$ & $95 \% \mathrm{CI}$ & SE & $p$ \\
\hline a path & & & & & .117 & $.063, .171$ & .028 & $<.001$ \\
\hline b path & & & & & .013 & $-.012, .037$ & .013 & .314 \\
\hline$c^{\prime}$ path & & & & & -.005 & $-.045, .036$ & .021 & .821 \\
\hline \multirow[t]{3}{*}{ Indirect effect $\left(a^{*} b\right)$} & & & & & .001 & $-.001, .004$ & .002 & .328 \\
\hline & \multicolumn{8}{|c|}{ Predicting Well-being } \\
\hline & \multicolumn{4}{|c|}{ Sample A $(\mathrm{N}=696)$} & \multicolumn{4}{|c|}{ Sample B (N=545) } \\
\hline Model Paths: & $\mathrm{b}$ & $95 \% \mathrm{CI}$ & $\mathrm{SE}$ & $p$ & $\mathrm{~b}$ & $95 \% \mathrm{CI}$ & SE & $p$ \\
\hline a path & .315 & $.239, .390$ & .038 & $<.001$ & .391 & $.312, .470$ & .040 & $<.001$ \\
\hline b path & .377 & $.321, .433$ & .029 & $<.001$ & .335 & $.278, .392$ & .029 & $<.001$ \\
\hline c' path & .058 & $.006, .111$ & .027 & .030 & .080 & $.027, .132$ & .027 & .003 \\
\hline Indirect effect $\left(a^{*} \mathrm{~b}\right)$ & 119 & $.085, .152$ & .017 & $<.001$ & .131 & $.096, .165$ & .018 & $<.001$ \\
\hline \multirow[b]{2}{*}{$\begin{array}{l}\text { Cross-lag Panel } \\
\text { Mediation Model Paths: }\end{array}$} & \multicolumn{4}{|c|}{ Sample A Weekly $(\mathrm{N}=530)$} & \multicolumn{4}{|c|}{ Sample A Monthly $(\mathrm{N}=518)$} \\
\hline & $\mathrm{b}$ & $95 \% \mathrm{CI}$ & $\mathrm{SE}$ & $p$ & $\mathrm{~b}$ & $95 \% \mathrm{CI}$ & SE & $p$ \\
\hline a path & .160 & $.105, .214$ & .028 & $<.001$ & .117 & $.063, .172$ & .028 & $<.001$ \\
\hline b path & .057 & $.016, .099$ & .021 & .007 & .008 & $-.011, .027$ & .010 & .396 \\
\hline$c^{\prime}$ path & .056 & $-.004, .117$ & .031 & .069 & .024 & $-.006, .054$ & .015 & .116 \\
\hline Indirect effect $\left(\mathrm{a}^{*} \mathrm{~b}\right)$ & .009 & $.002, .016$ & .004 & .015 & .001 & $-.001, .003$ & .001 & .405 \\
\hline
\end{tabular}

Note. *Future health behaviors were not assessed in the weekly assessments. 